\title{
EFFICIENT STAGNATION AVOIDANCE FOR MANETS WITH LOCAL REPAIR STRATEGY USING ANT COLONY OPTIMIZATION
}

\author{
Sharvani G S, Dr. A G Ananth and Dr T M Rangaswamy \\ R V College Of Engineering Mysore Road, Bangalore 560059 \\ sharvanim@yahoo.com, antisro@yahoo.com, rangaswamytm@rvce.edu.in
}

\begin{abstract}
Wireless networks such as Mobile AdHoc Networks (MANETs) have many advantages compared to wired networks. In MANETs the communication is not limited to a certain geometrical region. Swarm Intelligence based ACO algorithms provide interesting solutions to network routing problems. ACO based routing in MANETs will enhance the reliability and efficient packet delivery. They help in reducing control overhead due to their inherent scalable feature. The similarity between ant and nodes, colony and Wireless network helps to use ACO based routing in MANETs. The Termite Algorithms contains several tunable parameters and methods to automate the selection of optimal routes for different network conditions. However, Termite doesn't contain methods for determination of QoS, Route Maintenance; Load balancing etc. The present work focuses on development of an efficient routing algorithm "Modified Termite algorithms" (MTA) for MANETs. The MTA developed by adopting efficient pheromone evaporation technique will address to load balancing problems. By including QoS, efficient route maintenance, local repair strategy by prediction of node failures, the MTA is expected to enhance the performance of the network in terms of throughput, and reduction of End-to-end delay and routing overheads. The results of the analysis are presented in the paper.
\end{abstract}

Keywords

MANET, Swarm Intelligence, Ant Colony Optimization, Stagnation, QoS

\section{INTRODUCTION}

Due to dependency on Telecommunications networks for information processing, network resiliency has become a critical research area. Network resiliency helps in judging the ability of the network to support Quality of Service (QoS) to customers even in highly dynamic topology where the link/node failure is very frequent. Network Resiliency includes methodologies like prediction based route repair and efficient utilization of spare resources. These two methodologies guarantee seamless communication upon failure. Most of the failures are 
difficult to predict and eliminate, but it is possible to reduce the impact of set of specific failure by introducing network resilience techniques into the network design phase.

Wireless networks such as Mobile AdHoc Networks (MANETs) have many advantages compared to wired networks. In MANETs the communication is not limited to a certain geometrical region. Swarm Intelligence based ACO algorithms provide interesting solutions to network routing problems. ACO based routing in MANETs will enhance the reliability and efficient packet delivery. They help in reducing control overhead due to their inherent scalable feature. The similarity between ant and nodes, colony and Wireless network helps to use ACO based routing in MANETs.

Proposed work focuses on the development of a robust Swarm Intelligence system for MANETs. However due to certain challenging characteristic of MANETs; it is vulnerable to lose packets or gets congested. The main causes for these reasons are Dynamic nature of the topology. Due to mobility of nodes in MANETs routes between source and destination has to be adjusted dynamically. Therefore route maintenance and route discovery modules have to be very efficient. These two modules should help MANETs to discovery path to the destination and find valid routed for broken routes quickly without. Many approaches where found as discussed in Literature survey on LL).

The Termite Algorithms contains several tunable parameters and methods to automate the selection of optimal routes for different network conditions. However, Termite doesn't contain methods for determination of QoS, Route Maintenance; Load balancing etc.

An algorithm Modified Termite Algorithm (MTA) is proposed with a prediction mechanism that anticipates link breaks, and repairs them before they break, thereby avoiding unnecessary warning messages and reducing control overhead. Segmentation of data packets is provided, that need to be transmitted from the source to the destination i.e. transmission of data is done through an alternative path to the destination which had not been transmitted earlier prior to link failure through the failed route. Algorithm also provides QoS for providing best effort delivery and transmits its data only through those paths which can satisfy the required bandwidth. By including QoS, efficient route maintenance, and local repair strategy by prediction of node failures, the MTA is expected to enhance the performance of the network in terms of throughput, and reduction of End-to-end delay and routing overheads.

\subsection{Mobile Ad-hoc Networks (MANETs)}

Wireless communications can be categorized into Infrastructure dependent and infrastructure independent networks. The Cellular networks are infrastructure dependent where as Ad hoc Wireless Networks (AWN) are capable of operating without any fixed infrastructure support. MANET is one such category of AWN, which has a set of mobile agents, communicating with each other with-in radio frequency range. Since radio frequency range is limited, the communication traffic has to be relayed over several intermediate nodes. Therefore, MANET is also known as Multi Hop ad hoc networks. The functionality of the nodes is not only to fulfill as a host, but also as a router to forward packets to other nodes. Routing in MANET is a challenging task due to characteristics like node mobility - which leads to dynamic Topology, Error prone shared channel, channel contention and limited device availability. Hence, an efficient routing protocol has to be developed which is capable of reducing control overhead and utilizes the bandwidth efficiently [1] [2]. Ant Colony Optimization (ACO) is Swarm Intelligence (SI) based routing algorithm proposed for high quality routing in MANET. 
International Journal of Distributed and Parallel Systems (IJDPS) Vol.3, No.5, September 2012

\subsection{Ant Colony Optimization (ACO)}

ACO was inspired by an algorithm called "Ant System (AS)" [3]. ACO deals with artificial systems that are inspired from food foraging behavior of real ants, which can find optimal solutions in highly dynamic environment. A major problem with ACO algorithm is "stagnation". This occurs when all ants try to follow the same path to reach the destination (since there is more pheromone). This when applied in MANETs comes to a convergence state (equilibrium) and attracts all the data packets to follow the same path, which leads to congestion.

To mitigate the above problem of ACO algorithms, Pheromone control technique in the form of controlled evaporation for efficient routing is adopted.

Wireless networks such as MANETS have many advantages compared to wired networks. In MANETs the communication is not limited to a certain region (as in cellular region). MANETs have some peculiar routing challenges. Swarm Intelligence (SI) based routing algorithms provides an interesting analogy to the world of biology. SI based ACO algorithms provide interesting solutions to network routing problems. ACO based routing in MANETs will enhance the reliability and efficient packet delivery. They help in reducing control overhead due to their inherent scalable feature. The similarity between ant and nodes, colony and Wireless network helps to use ACO based routing in MANETs. Termite is one such ACO based algorithm which performs better than the AdHoc On demand Distance vector (AODV) [4].

\subsection{Related Work}

There has been a variety of research which contributed in the study of biologically inspired routing algorithms for MANETs. Ant net was developed in which the network performance was enhanced by prioritizing the packets. It was further enhanced in which features such as Techniques for Scalability, Enhanced pheromone methodology were adapted. The deposition of pheromone can be fine tuned based on function which decides source distance, Link quality, congestion and its velocity. Adopting these features, Ant net provides attractive features of Distributed control, Load balance etc.[5] [6].

Another technique is in which ant explores the network in a restricted broadcast behavior. It travels towards higher pheromone concentration if and only if there is no unvisited neighbor node entry (using binary value to indicate visited or not in the row and column) in the routing table.

Blocking-expanding ring search and Local Retransmission technique for routing in MANETs is one more technique which uses separate packets to form the paths. This helps the MANETs to perform well with minimum control packet overhead. To address shortest path problem with multiple source and destination, many meta-heuristics algorithms were used.

MANETs has limited resources like bandwidth, battery life etc, ACO based algorithm help MANETs in efficient resource utilization [7] [8]. Some striking features included by ACO algorithms are - Node entry to sleep mode when pheromone values in the routing table reach lower threshold, Omni directional antennas to adopt minimum energy broadcasting, efficient pheromone update methodology. These help MANETs manage battery power efficiently [9] [10] [11] [12] [13]. 
International Journal of Distributed and Parallel Systems (IJDPS) Vol.3, No.5, September 2012

\subsubsection{QoS in MANETS}

Quality of service (QoS) is service level guarantee given by the network providers to the requesting user. There were many QoS based routing using SI. In which few aimed at best effort delivery, where as others provided real time delivery. The main aim of best effort delivery was to have efficient packet delivery ratio, whereas real time delivery aimed at less end-to-end delay and less routing overhead. Few of the papers describing QoS algorithms in ACO are [14][15][16][17] [18].

\subsubsection{Local Route repair strategy using prediction}

Local route repair becomes an important concept in MANET since it reduces control overhead. This makes MANETs for efficient routing. Prediction is based on the mobility and cache [19]. AODV improved its route failure and quality of service with the help of GPS [20].Predictive preemption based route repair strategy, proves that AODV provides efficient packet delivery ratio, reduces end-to-end delay and reduces the control overhead [21].TCP based feedback mechanism which tells the source to freeze and stop sending the packets until it gets the route reestablishment notification from the node which found Link failure before. This enhances through put and avoids unnecessary packet delivery [22].

\subsubsection{Stagnation avoidance algorithms}

Elitist Ant System (EAS) algorithm is a variation of ACO [23]. Here pheromone deposits are only for the best path found. The major problem with this algorithm is early stagnation. Max-Min Ant systems (MMAS) [24] overcome the early stagnation problem by adding an extra constraint which says that the pheromone is bounded between maximum and minimum pheromone concentration.

Stagnation avoidance algorithm was developed while ACO found its application in Scheduling of flexible manufacturing systems [25]. The methodology developed deals with quick convergence and stagnation avoidance in which parameters of ACO such as evaporation co-efficient which control the trail and its visibility are fine tuned. MMAS was further improved by adopting Minimum Pheromone Threshold Strategy (MPTS)[26]. Here the bound between maximum and minimum threshold is fine tuned based on performance of the network. Improved Lower Limits for Pheromone Trails in ACO adopts improved estimates of the lower pheromone value. This helps algorithms like MMAS (sets implicit pheromone trail limit) to avoid stagnation [27]. Another work on stagnation avoidance for scheduling of real-time tasks [28] uses a non-preemptive scheduling approach based on distance function as an extra parameter in the transition rule with the pheromone information.

A framework for stagnation avoidance was developed while adapting ACO in TSP [29]. This frame work evaluates each solution against the last " $\mathrm{N}$ " solutions globally constructed by ants. As soon as "N" solutions are available, their moving average "A" is computed. New value of A is compared with old value. If New A is lower than old, the trail level of the last solution's moves is increased, otherwise it is decreased.

An ACO algorithm with improved pheromone correction strategy for the Minimum Weight Cover Problem (MWCP) [30] [31] explains that new Hybridization techniques. Corrections to the pheromone trails are performed based on suspicion whether it is good or bad. It decrements the pheromone trails very fast if it finds more suspicious elements. In Hybrid architecture, the age of the ant decides whether the trail should be increased or not [32]. Another solution for 
stagnation was found by using information entropy [33] which helped the algorithm to fine tune its pheromone trails for local search.

\section{Routing in MANETs with MTA}

Before routing the network has to stabilize itself by sending hello packets to its neighbors and build the routing or pheromone table. This helps them to know their neighbors. Neighbor nodes must be carefully handled as they can forward/ originate packets. However, they always give preference for packets to be forwarded. Thus, the neighbor row must be declared decayed before the neighbor node can be removed from the pheromone table. If a neighbor is determined to be lost by means of communications failure, the neighbor row is simply removed from the pheromone table. Similarly a decayed column indicates that no traffic has been seen which was sourced by that node.

\subsection{Routing table in MTA}

Termite's pheromone table is analogous to routing table. Each node in the network maintains a pheromone table which keeps track of percentage of pheromone in each neighbor link. The table consists of row representing the neighbors and columns correspond to destinations (except for node itself). Table size is dynamic and varies based on the destinations heard recently. An entry of node ' $\mathrm{n}$ ' in the table is referenced by $P_{i, d}^{n}$ where $i \in N^{n}$ is the neighbor and 'd' denotes the destination where $d \in D^{n}$, in other words, $P_{i, d}^{n}$ is the percentage of pheromone from a node 'd' on the link with neighbor 'i' at node 'n'.

\subsection{Pheromone update/Decay}

Pheromone is updated only by data packets as compared to other ACO based algorithm in which all forward ants, backward ants and data packets update pheromone concentration. When a packets arrives at a node ' $n$ ' from source $s$ and a previous hop 'p', the new pheromone concentration entry for $P_{p, s}^{n}$ for the source of the packet is increased by constant pheromone value ' $\gamma$ ' equation as shown in Eqn (1) the.

$$
P_{p, s}^{n} \leftarrow P_{p, s}^{n}+\gamma \ldots \ldots \ldots \ldots \ldots \ldots . . . \text { Eqn (1) }
$$

Pheromone decay is an important factor for efficient packet delivery, if not handled properly, it leads to stale entries in the network which lead to packet drops or it might lead to improper load in the network due to data packets travelling on the optimal path and increasing the pheromone concentration. This tells other packets to travel blindly not knowing whether the link is overloaded or not.

Pheromone values in the routing table will be decayed periodically as shown in Eqn (2) by multiplying it with decay factor, $\mathrm{e}^{-\tau}$ where $\tau \geq 0$ and is a global parameter. There should be a balancing act between the pheromone decay process i.e. neither too quick nor slow. The nominal pheromone decay interval is one second which is called as decay period.

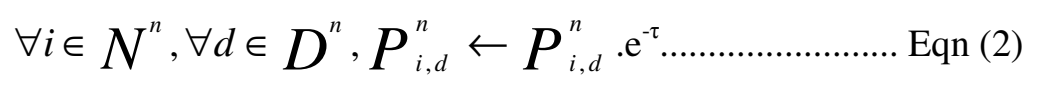


If all the pheromone for a particular node (as it has not received any packets for a long time) decays, the row for that is removed from the routing table. However, column entry cannot be deleted directly if it is also neighbor node. Pheromone bounds like max, min and initial pheromone values (prevents extreme difference in pheromone value) in the routing table plays a major role in stagnation problems. Table updates new column and row for the neighbor as it can also be a destination. When a packet is received from unknown source then the new entry for that node is created on the routing table. If the nodes are just destinations then only the column are created[34].

For example ,Source 'S' considers N1, N2 and N3 as neighboring nodes since they are within the transmission range. Once the neighboring nodes are known then the routing table is updated as shown in Table 1(route discovery also helps in updating the routing table). When node ' $\mathrm{S}$ wants to communicate with destination ' $\mathrm{D}$ ' it checks for the highest trail for destination 'D' and then forwards the packet.

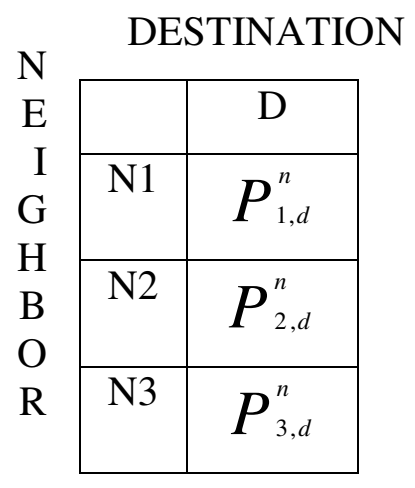

\section{TABLE 1 ROUTING TABLE AT NODE 'S'}

\section{Efficient Stagnation avoidance Technique}

Out of the many decay functions analyzed, the exponential decay techniques in ACO are more applicable for dynamic networks like MANETs. The limitation of this technique is pheromone evaporates (uniformly) very quickly after some time leaving no traces for the data transmission. Hence, fresh route discovery is established to know the destination address. This causes more control overhead and makes bandwidth inefficient. To deal with this problem, controlled exponential evaporation is adopted which fine tunes evaporation based on the stability factor ' $\Delta$ ' of the node.

Each node in the network also maintains the Node stability Table for all its neighboring nodes. An example is shown in TABEL2. This table helps in finding more stable nodes. 
International Journal of Distributed and Parallel Systems (IJDPS) Vol.3, No.5, September 2012

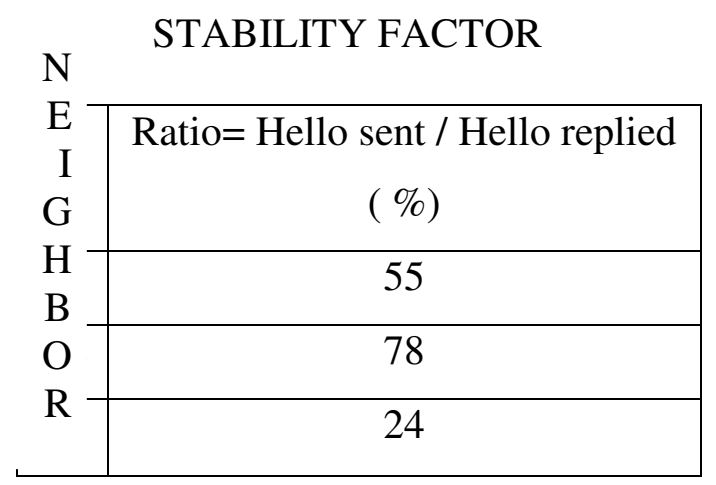

\section{TABLE 2 NODE STABILITY FACTOR OF NEIGHBOR NODES AT NODE 'S'}

Evaporation is fine tuned based on the stability factor of the node in the vicinity of the node that forwards the packet. During packet forwarding time, each node calculates the 'hello' sent and 'hello' received by its neighboring nodes and calculates the stability ratio shown in Eqn (3)

$$
\text { Ratio }=(\text { Hello Replied } / \text { Hello Sent }) * 100 \ldots \ldots \ldots \ldots \ldots \ldots \ldots \ldots \text {............ (3) }
$$

With the help of ratio calculated, evaporation is controlled for each neighboring node by fine-tuning the decay speed like decaying fast for unstable nodes and decaying little slower for stable nodes.

Exponential Decay may result in faster lost of pheromone trail traces. Pheromone trail is controlled by adding an extra parameter ' $\Delta$ ' to the exponential decay factor.

$$
\text { Where } \Delta= \begin{cases}0.003 & \text { If Ratio is }>75 \% \\ 0.002 & \text { If Ratio is } \leq 75 \% \text { and }<55 \% \\ 0.001 & \text { If Ratio is } \leq 55 \%\end{cases}
$$

This technique helps the MANETs to overcome stagnation problem, it also reduces the control overhead and have efficient packet delivery ratio. ' $\Delta$ ' is tunable based on the application and the node mobility as shown in Eqn 4. This tells with what percentage the link should decay its pheromone (tunable). Table 3 describes pheromone decay factor vs ratio.

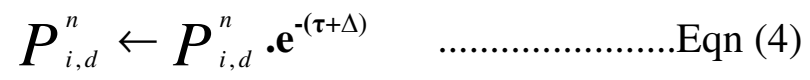

\begin{tabular}{|c|c|c|c|}
\hline & $\begin{array}{c}\text { If Ratio } \\
\geq 75 \%\end{array}$ & $\begin{array}{c}\text { If Ratio } \\
<75 \% \text { and } \geq 55 \%\end{array}$ & $\begin{array}{c}\text { If Ratio } \\
<55 \%\end{array}$ \\
\hline Decay Factor $\mathrm{e}^{-(\tau+\Delta)}$ & $\left(\mathrm{e}^{-\tau+0.003}\right)$ & $\left(\mathrm{e}^{-\tau+0.002}\right)$ & $\left(\mathrm{e}^{-\tau+0.001}\right)$ \\
\hline
\end{tabular}

TABLE 3 DECAY FACTOR FOR DIFFERENT RATIO 
Exponential Decay may result in faster lost of pheromone trail traces. Pheromone trail is controlled by adding an extra parameter ' $\Delta$ ' to the exponential decay factor. Decay should be efficiently handled (at an interval of one second); otherwise a high decay rate will quickly reduce the amount of remaining pheromone, while a low value will degrade the pheromone slowly[35].

A source ready to forward packet to the destination, takes packet forwarding equation's help to find out the next hop neighbor. This formula (as shown in Eqn (5)) updates pheromone value for that destination' $d$ ' on each outgoing link ' $i$ '. The random selection of next neighbor is performed with the help of packet forwarding equation. Extra care must be taken by not forwarding packet to the previous hop ' $\mathrm{p}$ '.

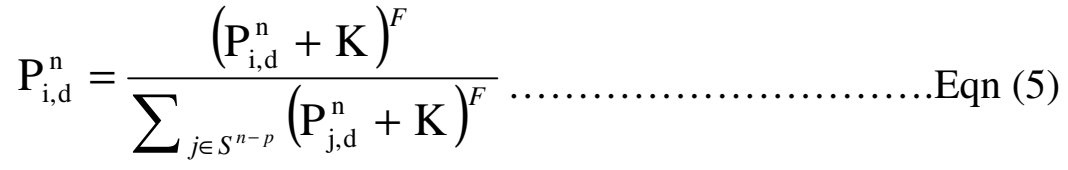

Where F (pheromone sensitivity) and $\mathrm{K}$ (pheromone threshold) are tunable based on their sensitivity to amount of pheromone values.

\section{MTA providing QOS and Efficient route repair strategy}

The application has three phases which Route Discovery, Route Maintenance and Route Failure Handling .The route discovery is responsible for providing Quality of Service (QoS) between a given source and a given set of reachable destination. Route Request (RREQ) performs a walk over the network until it reaches the requested destination. If the bandwidth is available then the route request packet is forwarded towards the destination node. While traveling to the destination the Route Request collects the available capacity of each link, the number of hops visited. Then it will respond with a Request Reply (RREP) packet, which will be initialized with the available bandwidth value.

Route maintenance phase as shown in Fig 1, basically helps in strengthening the route which has already been established during the route discovery phase. The strengthening of the route is done by the data packets. Every time a data packet travels a particular link, it increases the pheromone value of that link in the respective nodes. This phase not only strengthen the path but also performs the periodic decay (above technique) of the established path in order to search for a new optimal path which might have changed due to mobility of nodes. This part of the phase gives a dynamic nature to the algorithm. 


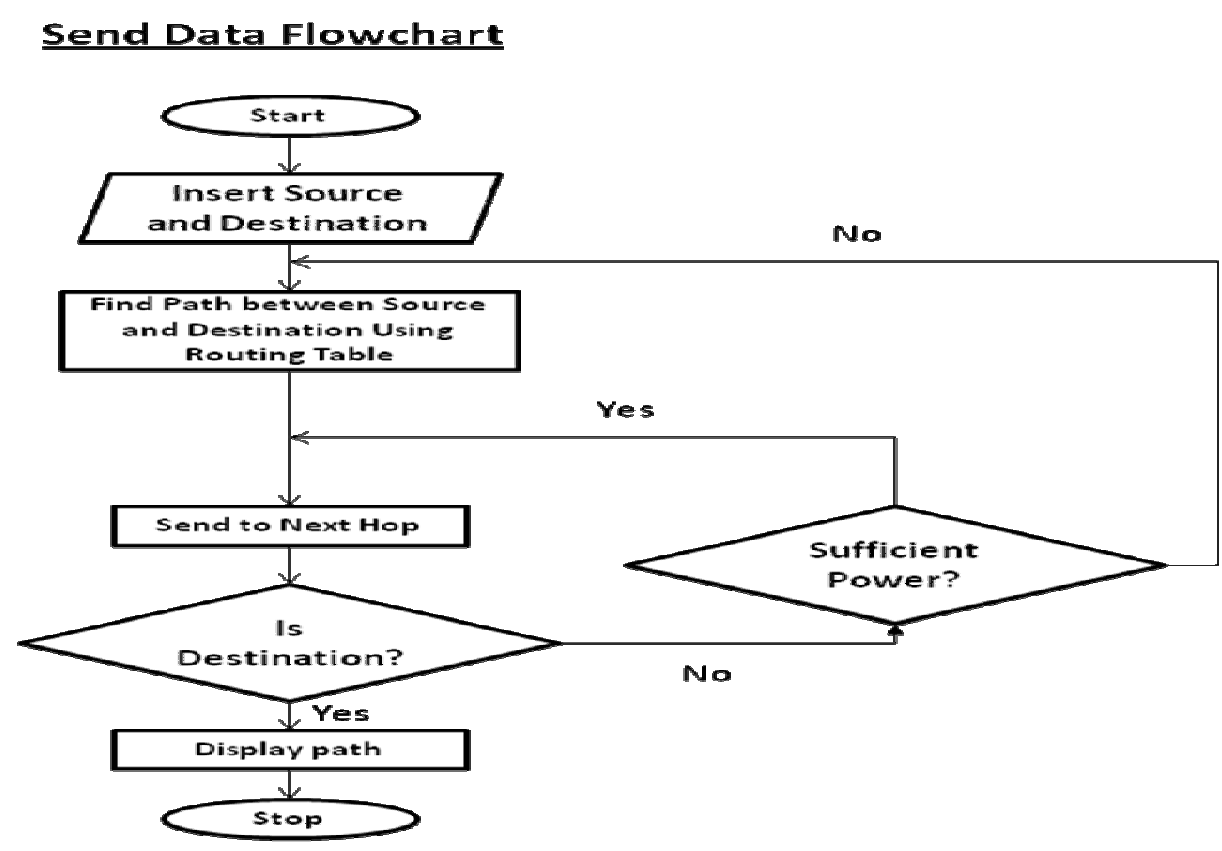

Fig 1: Route discovery Phase

Nodes in MANETs may crash due to battery failure. Such failures are handled by the route failure handling phase. The detection of route failure is predicted through the Lagrange's interpolation methodology. Here a node calculates battery threshold (P) using Lagrange's interpolation methodology, if the battery threshold is reached, then source node preempts the transmission and bypass the packets to the next optimal path [36].

\section{Predict ()}

$\{$ If $(\mathrm{P}<=$ Min_Acc_Power) then

$\{$ Send_warning (predecessor_node);

Segmentation_Of_Packets ();

//transmit only the remaining data packets which had not been transmitted earlier //through this route, prior to link failure, through an alternative route, to the //destination\}

\section{RESULTS AND DISCUSSION:}

Some of the improvements to Termite algorithms could be fine tuning pheromone values, reserving resources in the form of time slots, bandwidth and efficient local route repair strategy. The exponential pheromone decay technique based on node stability is adopted which makes the MANET efficient in terms of control overhead, bandwidth, packet delivery etc. 
The present work focuses on development of an efficient routing algorithm Modified Termite Algorithm (MTA) using termites based on ACO and implementation on MANET. The primary metrics considered for the algorithm are Throughput, End-to-End delay and routing over head against the faulty nodes.

The behavior of success rates with the number of termites (nodes) has been studied . It is considered as appropriate to choose the number of termites (Nodes) 30 for determining the throughput, end-to-end delay and control overheads for the MANET. Using MTA with QoS and with effective Local route repair strategy detailed analysis has been carried out on MANETs to determine the Throughput, end-to-end delay and routing overhead by varying the number of faulty nodes.

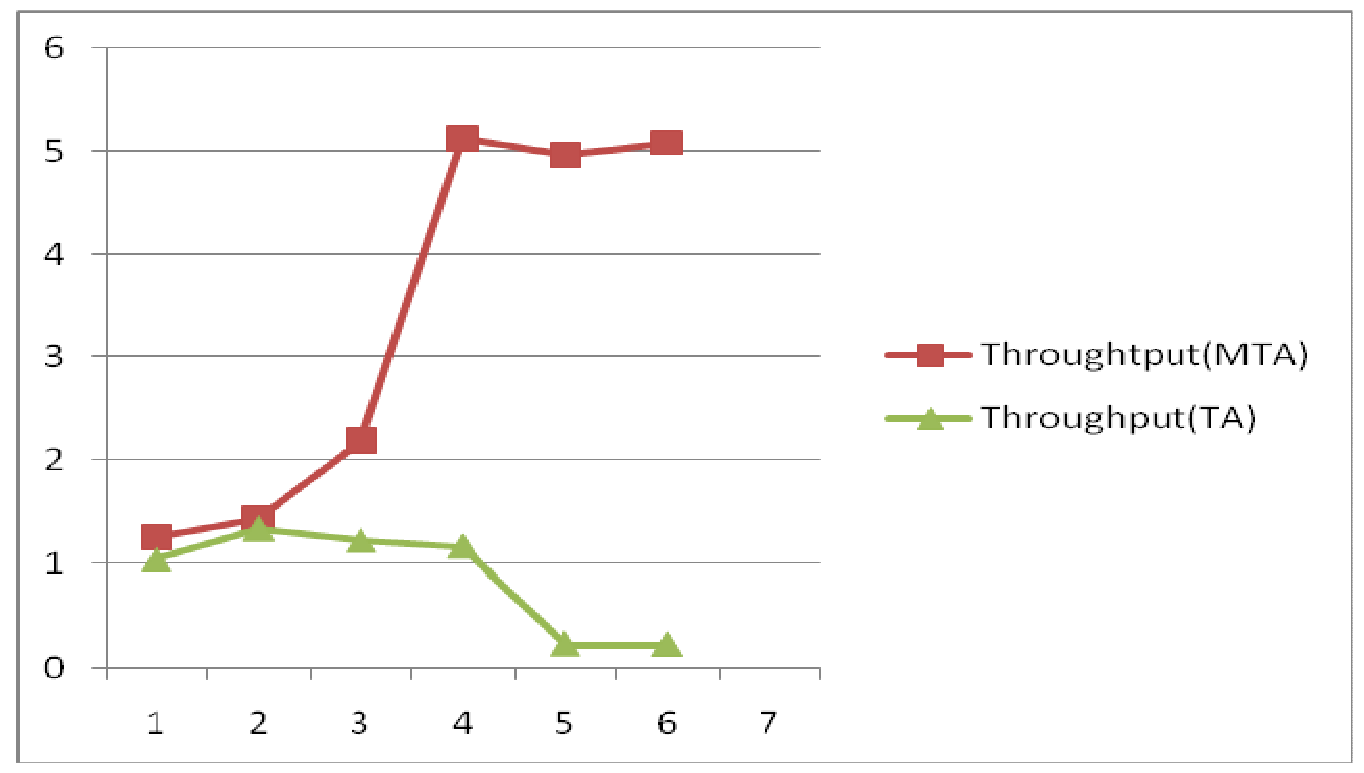

Fig 2 Throughput (Mbps/sec) Vs No of Faulty Nodes

The variation of throughput with the increase of Faulty nodes between 1-6 determined using ACO based MTA is shown in Fig 2. The figure clearly demonstrates that the MTA based MANET shows continuous increase in throughput $\sim 4.16$ times with the faulty nodes, where as the throughput for termite algorithm remains low $<1.2$ and show very small variation with increase in faulty nodes. This suggests that the MTA algorithm implemented on a MANET with 30 nodes indicates large improvement in the throughput $\sim 76 \%$ with the increase in the faulty nodes as compared to termite algorithms.

The MTA implemented on MANET has been used to determine the end-to-end delay with the increase of Faulty nodes between 1-6. Fig 3 shows the end to end delay variations for increase of Faulty nodes between 1-6. In the same figure the end to end delay variations for the termite algorithm which does not incorporate efficinet pheromone decay technique is also shown for comparison. 


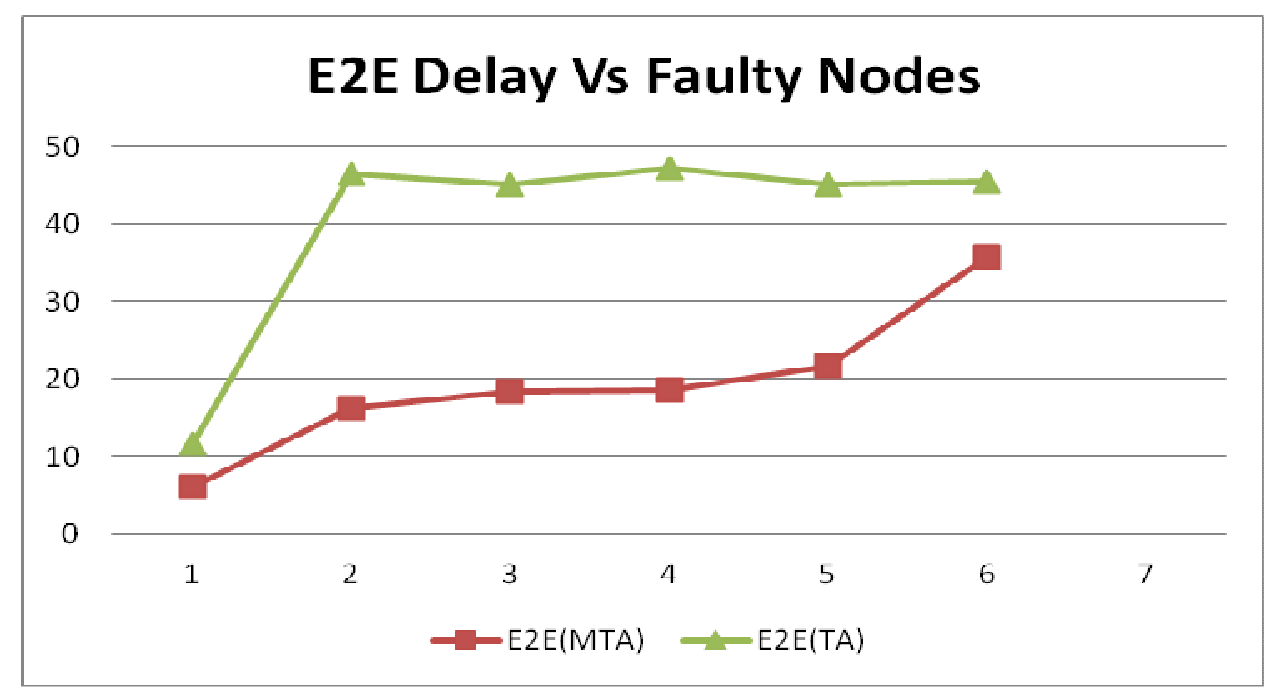

Fig 3 End to End Delay (sec) Vs No of Faulty Nodes

It is seen form the figure 3 that the end to end delay for termite algorithm is $\sim 4.5$ times increase in time delay with increase of Faulty nodes between 1-6. Where as the end to end delay variation for MTA algorithm $~ 4.75$ show significant variations with increase of Faulty nodes. This suggests that the MTA algorithm implemented on a MANET with 30 nodes shows reduction in the end-to-end delay $\sim 22 \%$ with the increase of Faulty nodes as compared to termite algorithms.

The MTA based MANET has been used to determine the routing over head changes with the increase of Faulty Nodes. Routing overhead is the ratio of number of control packets required for delivery of data .Fig 4 shows the routing over head changes for increase of Faulty Nodes between 1-6. In the same figure the routing over head changes for the termite algorithm is shown for making a comparison. 
International Journal of Distributed and Parallel Systems (IJDPS) Vol.3, No.5, September 2012

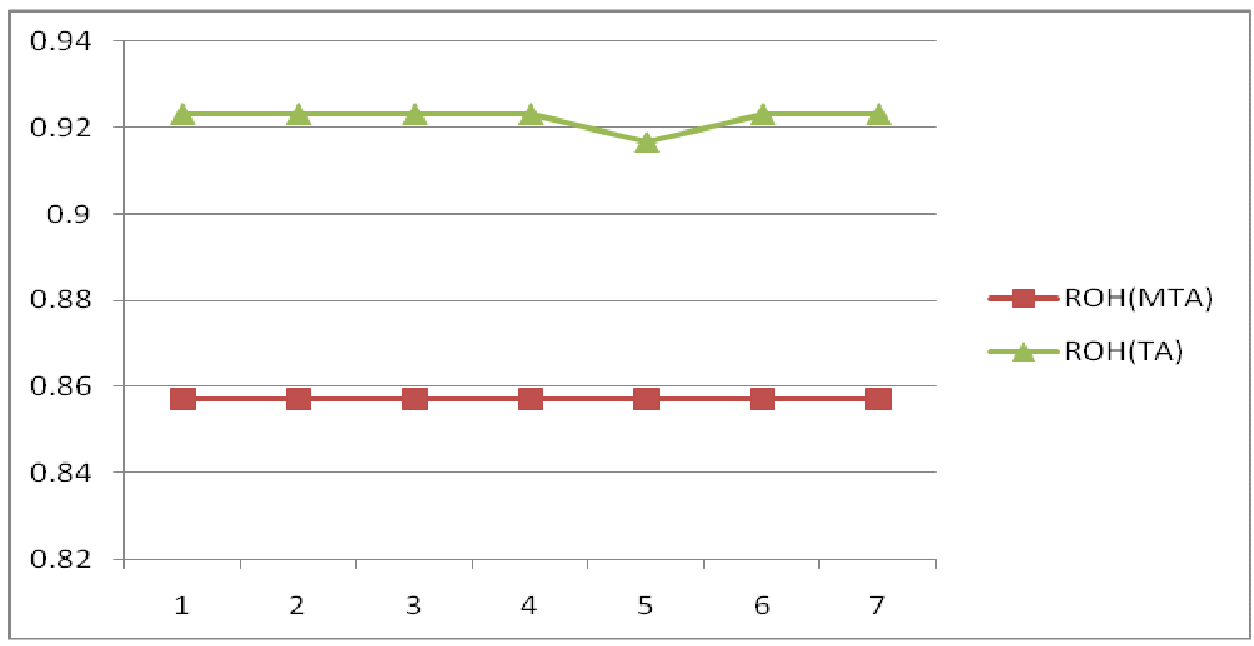

Fig 4 Routing Overhead (packets) Vs No of Faulty Nodes

The Fig 4 clearly demonstrates that for termite algorithms the Routing overheads $\sim 0.1$ times increase with the increase of Faulty Nodes. Where as the routing overheads increases for MTA algorithm is constant and do not change with increase of Faulty Nodes This suggests that the MTA algorithm implemented on a MANET with 30 nodes shows reduction in the overheads $\sim 9 \%$ with increase of Faulty Nodes as compared to termite algorithms suggesting that MTA algorithms shows improvement in routing overheads compared to termite algorithms.

The results of the analysis suggests that the stagnation problems can be overcome by fine tuning of the Pheromone concentration based on node stability factor. The modified Termite algorithm (MTA) with fine tuning of pheromone concentration shows significant increase in the throughput, large decrease in end to end delay and small decrease in routing overheads. The efficient route discovery can improve the QoS by efficient bandwidth utilization. The reduction of routing overheads can be used for achieving the local route repair strategy

\section{CONCLUSION}

From the analysis carried out, the following conclusions are drawn

1. The stagnation problems can be overcome by fine tuning of the Pheromone concentration based on node stability factor.

2. The MTA implemented on MANET is found to perform better for 30 nodes as the success rates are found to be maximum beyond this range.

3. The MTA implemented on MANET with fine tuning of pheromone concentration shows significant increase in the throughput $\sim 76 \%$ with increase of Faulty Nodes 1-6 compared to termite algorithms 
International Journal of Distributed and Parallel Systems (IJDPS) Vol.3, No.5, September 2012

4. The MTA implemented on MANET shows significant decrease in End-to-end time delay 22\% with increase of Faulty Nodes 1-6 compared to termite algorithms

5. The MTA implemented on MANET shows a small decrease $\sim 9 \%$ in the routing overheads with increase of Faulty Nodes 1-6 and indicates improvement in routing overheads compared to termite algorithms

\section{REFERENCES}

[1]. C S R.Murthy and B S Manoj, “ Ad Hoc Wireless Networks : Architecture and protocols” prentice Hall PTR, 2004

[2]. Elang Huang, Wenjun Hu Jon, Crowcroft Lan, “ Towards commercial Mobile Ad Hoc Network applications: A Radio Dispacth System", MobiHOC May, 2005,Illinois, USA.

[3]. March Dorigo," Ant Colony Optimisation and swarm intelligence", 4th International workshop, ANTS, 2004, Brussels, Belgium, Sep 2004( Proceedings in LNCS, Berlin:Springer)

[4]. Martin Roth and Wicker S, “ Asymptotic pheromone behavior in Swarm Intelligent MANETs", sixth IEEE Interntional conference on mobile and wireless communication network, Berlin:springer, 2004, pp 335-346

[5]. Claudio E Torres, Louis F Rossi, Jeremy Keffer, Ke Li and Chien Chung Shen," Modelling analysis and simulation, of ant based networking routing protocols", Springer science Business Media, LLC, 2010.

[6]. Florino De Rango and Annalisa Socievole ," Meta-Heuristic Techniques and Swarm Intelligence in MANETs",Mobile ad Hoc Networks:Application,, Jan 2011, pp 245-264

[7]. Ahmed A, A Radwan, Tarek M Mahmoud and Essam H Hussein," AntNet-RSLR: A Proposed Ant Routing Protocol for MANETs", SIECPC, April 2011, pp 1-6.

[8]. Martin Roth and Wicker S, “ Asymptotic pheromone behavior in Swarm Intelligent MANETs", sixth IEEE Interntional conference on mobile and wireless communication network, Berlin:springer, 2004, pp 335-346

[9]. Gunes M, Kahmer M and Bouazzi I," Ant Rouitng Algorithm (ARA) for MANETs-New features and results", The Secong Mediterrian workshop on Ad Hoc Networks, 2003.

[10]. Daneshlab, P Vrancx and A Nowe," Using Pheromone repulsion to find disjoint paths", 5th International workshop, ANTs,2006.

[11]. Wong L H and Looi C K “ Swarm Intelligence- New techniques for adaptive systems to provide learning support”, Interactive learning Environments, Publised online-2010.

[12]. Dorigo Marco , Birattari, Mauro and Blum, Christian and Clerc, “ Ant Colony Optimisation and Swarm Intelligence", 6th international Conference, ANTS 2008, Springer Berlin/ Heidelberg, LNCS, Vol 5217, 2008, pp 25-36

[13]. Kriesel, David and Cheung, Eugene and Sitti, Metin and Lipson," Beanbag Robotics: Robotic Swarms with 1-Dof Units", " Ant Colony Optimisation and Swarm Intelligence”, 6th international Conference, ANTS 2008, Springer Berlin/ Heidelberg, LNCS, Vol 5217, 2008, pp 22-24

[14]. S Zouaidi, A Mellouk, M Bourennane, S Hoceini," Design and performance analysis of inductive QoS scheduling for dynamic network routing", 16th International Conference on Software Telecommunications and Computer networks, 2008, pp 140-146 
International Journal of Distributed and Parallel Systems (IJDPS) Vol.3, No.5, September 2012

[15]. Yang Hao and Qin Zhiquang," Chaos ant colony optimization algorithm for the multi-QoS constraint multicast routing problem", ICCCAS, 2010, pp 190-192

[16]. H Shokrani and S Jabbehdari," A Novel ant based QoS routing for Mobile Ad Hoc Metworks", ICUFN -09, USA, IEEE Press, 2009,pp79-82

[17]. M Shamim Hossain and Abdulmotaleb Saddik " QoS requirement in the multimedia transcoding service selection process", IEEE transactions on Instrumentation and Measurement, Vol 59, No 6, June 2010, pp 1498-1506

[18]. Fidanova S, Atanassov K and Marinov," Start strategies of ACO applied on Subset Problems", Numerical Methods and Application, LNCS, Springer, Germany, 2011, pp 248-255.

[19]. A. Cahill et al., "Link cache extensions for predictive routing and repair in ad hoc wireless networks", in Proceedings of the 4th IEEE Conference on Mobile and Wireless Communication Networks (MWCN), September 2002, pp. $43-52$.

[20]. S. Crisòstomo et al., "Improving AODV with Preemptive Local Route Repair", Technical Report Series:DCC-2003-0, Universidade do Porto, Portugal, 2003.

[21]. Sofiane Boukli Hacene, Ahmed Lehireche, Ahmed Meddahi" Predictive Preemptive Ad Hoc On-Demand Distance Vector Routing Evolutionary Engineering and Distributed Information Systems Laboratory, EEDIS, Computer Science Department, Sidi Bel Abbes University, 2006.

[22]. W. J Su et al., "Mobility Prediction and Routing in Ad hoc Wireless Networks", International Journal of Network Management, Wiley \& Sons, 2000.

[23]. Ducatelle F, Di Caro G and Gambardella L M, "Principles and applications of Swarm Intelligence for adaptive routing in telecommunications networks", Swarm Intelligence, 2010.

[24]. T Stutzle, H H Hoos,"Max-Min Ant system” Future Generation Computing Syst , PP 889914,2000 .

[25]. R Kumar, M K Tiwari and R Shankar, "Scheduling of flexible manufacturing systems: an ant colony optimization approach", Proceedings Instn Mech Engrs Vol 217, Part B: J Engineering Manufacture, ,pp 1443-1453,2003.

[26]. Kuan Yew Wong, Phen Chiak See, “ A New minimum pheromone threshold strategy(MPTS) for Max-min ant system “, Applied Soft computing, Vol 9, pp 882-888, 2009

[27]. David C Mathew, “ Improved Lower Limits for Pheromone Trails in ACO”, G Rudolf et al(Eds), LNCS 5199, pp 508-517, Springer Verlag, 2008.

[28]. Laalaoui Y, Drias H, Bouridah A and Ahmed R B, “ Ant Colony system with stagnation avoidance for the scheduling of real time tasks", Computational Intelligence in scheduling, IEEE symposium, pp 1-6,2009.

[29]. E Priya Darshini, " Implementation of ACO algorithm for EDGE detection and Sorting Salesman problem",International Journal of Engineering science and Technology, Vol 2, pp 23042315,2010

[30]. Alaa Alijanaby, KU Ruhana Kumahamud, Norita Md Norwawi, "Interacted Multiple Ant Colonies optimization Frame work: an experimental study of the evaluation and the exploration techniques to control the search stagnation", International Journal of Advancements in computing Technology Vol 2, No 1, pp 78-85,March 2010 .

[31]. Raka Jovanovic and Milan Tuba, "An ant colony optimization algorithm with improved pheromone correction strategy for the minimum weight vertex cover problem", Elsvier, Applied Soft Computing, PP 5360-5366, 2011. 
International Journal of Distributed and Parallel Systems (IJDPS) Vol.3, No.5, September 2012

[32]. Priyanka Sharma, Dr K Kotecha, "Optimization in stagnation avoidance of ACO based routing of Multimedia Traffic over Hybrid MANETs", International Journal of computer science and technology, IJCST, Issue 2, ISSN: 2229-4333(print), 0976-8491(online), 2011

[33]. Zar Ch Su Hlaing, May Aye Lhine, "An Ant Colony Optimization Algorithm for solving Traveling Salesman Problem", International Conference on Information Communication and management (IPCSIT), Vol, 6, pp 54-59, 2011.

[34]. Martin Roth and Stephen Wicker "Termite: Emergent Ad-Hoc Networking " Wireless Intelligent Systems Laboratory School of Electrical and Computer Engineering Cornell University Ithaca, New York 14850 USA

[35]. Sharvani G S, Dr. Ananth G and Dr T M Rangaswamy, "Analysis of different pheromone decay techniques for ACO Based routing in Ad Hoc wireless Networks "( communicated)

[36]. Sharvani.G.S, Dr.T.M.Rangaswamy," Improving Qos for Ad-Hoc Wireless networks using Predictive premptive Local route repair strategy " International Conference on Computer Science and Information Technology(COSIT), Bangalore pp 55-61,Jan 2011 